\title{
FAST BEAM-ION INSTABILITY DRIVEN LONGITUDINAL DAMPING
}

\author{
Yujong Kim*, C. Kim, K. H. Kim, C. D. Park, M. H. Chun, J. S. Yang \\ I. H. Yu, M. Kwon, J. Y. Huang, S. H. Nam, M. H. Cho, W. Namkung, and I. S. Ko \\ Pohang Accelerator Laboratory, POSTECH, Pohang, 790-784, Korea
}

\section{Abstract}

In the Pohang Light Source storage ring, we empty 68 buckets of total 468 buckets to cure the conventional ion trapping. However, the fast beam-ion instability is occasionally generated at higher beam current. Immediately after the strong vertical beam shaking due to the fast beamion instability, the longitudinal coupled bunch mode instabilities are substantially damped down without any active feedback system. We have described the fast beam-ion instability driven longitudinal damping and its mechanism in the Pohang Light Source storage ring.

\section{INTRODUCTION}

The molecules of the residual gas in the vacuum chamber of an electron storage ring can be ionized by the Coulomb collisions with moving electron beams or by the synchrotron radiation. These ions may be trapped in the potential well of the negatively charged electron beams within a single turn (fast beam-ion instability, FBII) or over many turns (conventional ion trapping, CIT) [1]-[4]. Generally, the CIT can be controlled by introducing an ion clearing gap with several empty buckets in the filling pattern. Since there is no charged beam in the gap, ions will be overfocused and be free from the potential well in the region. The FBII is generated when the preceding bunches generate ions and the following bunches are perturbed by the ions within a single turn. Therefore, the FBII can not be cured by introducing a single ion clearing gap. Since the number of the generated ions is increased along the bunch train, the perturbed coherent beam oscillation is also increased along the bunch train [1]-[4]. Although the FBII generally generates the vertical beam instability and the vertical beam emittance growth, it can also make beams longitudinally stable under special conditions. Immediately after the strong vertical beam shaking due to the FBII, the longitudinal beam parameters such as the synchrotron oscillation frequency and the RF noise frequency are changed via the synchro-betatron coupling. Under properly changed longitudinal parameters, the longitudinal coupled bunch mode instabilities (CBMI's) are substantially damped down by the RF noise induced dipole mode parametric resonance of the phase modulation. We have described the FBII driven the longitudinal damping and its mechanism in the Pohang Light Source (PLS) storage ring by using the streak camera and the longitudinal feedback system (LFS) as data acquisition systems (DAS).

\footnotetext{
*yjkim@POSTECH.edu
}

\section{SYNCHRO-BETATRON COUPLING}

Since there is no global orbit feedback system in the PLS storage ring, and there is the RF noise-sidebands which generate the energy spread [5], the dispersion is none zero at the RF cavities. Moreover, we are operating the PLS storage ring under the horizontal chromaticity $\xi_{x}$ of 0.35 and the vertical chromaticity $\xi_{y}$ of 0.26 to control the transverse head-tail instability. In these cases, the closed orbit at the RF cavities moves as a function of energy, and the orbit of beams is displaced with respect to the closed orbit when they cross the RF cavities to supply the lost energy. Therefore, the phase of the betatron oscillation will be changed after successive turns according to that of the synchrotron oscillation [6]. Further, due to the none zero dispersion at the RF cavity, the betatron oscillation will change the orbit length per one revolution and the beam position with respect to the RF phase [6]. We call this phenomenon as the synchro-betatron coupling that the transverse beam motion is coupled by the longitudinal beam motion and vice versa [6]. The none zero chromaticity will enhance the synchrobetatron coupling and will be appeared as the betatron tune modulation which is given by

$$
\nu=\nu_{o}+\xi \nu_{o} \delta,
$$

where $\nu$ and $\nu_{o}$ are the modulated tune and the original one, $\xi$ is the chromaticity, and $\delta=\Delta p / p$ is the energy spread [6]. Actually, during the PLS normal operation period, we have occasionally observed a lower satellite around the betatron tunes via the synchro-betatron coupling due to the none zero chromaticity and the RF noise-sidebands in the No. 3 low level RF system. The satellite can be removed by reducing the RF noise-sidebands or by reducing the chromaticity to zero. When there is the RF noise-sidebands with the first harmonic frequency of about $5.5 \mathrm{kHz}$, we observed the lower satellite around the vertical betatron tune via the synchro-betatron coupling whose relation is $\nu_{y}-16 \nu_{s}=8$. Here $\nu_{y}$ and $\nu_{s}$ are the vertical tune of 8.17622589 and the synchrotron tune of 0.0109497 , respectively. Note that when $\nu_{y} \gg \nu_{s}$, beams can stay at enough stable tune space to oscillate safely though there is the synchro-betatron coupling [6].

\section{FAST BEAM-ION INSTABILITY}

Normally, the residual molecules in the PLS storage ring are mostly $\mathrm{H}_{2}(90 \%)$ and $\mathrm{CO}(5 \%)$. Although the ionization rate of $\mathrm{CO}$ is higher than that of $\mathrm{H}_{2}$, ions are generated mostly by $\mathrm{H}_{2}$ due to its much higher population. However, immediately after the installation of a new vacuum 

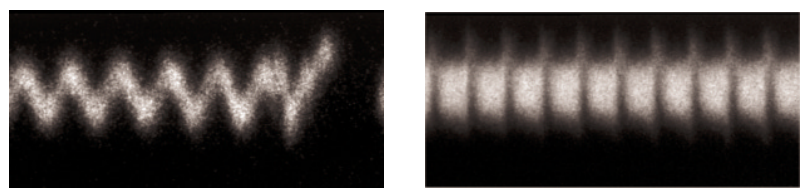

(a)

(b)

Figure 1: Streak camera images of the 400-bunch beam motion for one train (a) and ten trains (b) when the weak FBII is generated due to $\mathrm{CO}$ at $196.0 \mathrm{~mA}, 1.2$ nTorr. The maximum horizontal time scales are $1 \mu \mathrm{s}$ (a) and $10 \mu \mathrm{s}$ (b), respectively, and the maximum vertical time scale is $300 \mathrm{ps}$ for both cases.

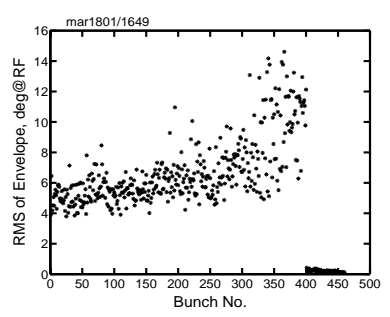

(a)

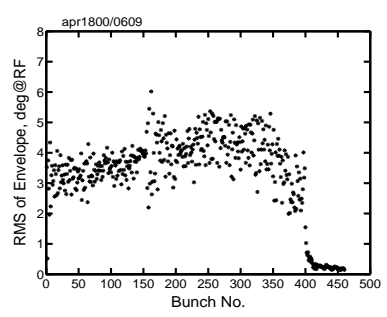

(b)
Figure 2: RMS synchrotron oscillation amplitudes along a bunch train which are simultaneously recorded by the LFS when the FBII is generated due to CO (a) at 196.0 $\mathrm{mA}, 1.2$ nTorr as shown in Fig. 1 and due to $\mathrm{H}_{2}$ (b) at $280.27 \mathrm{~mA}, 70.0$ nTorr as shown in Fig. 3.

component, the population of $\mathrm{CO}$ would be increased up to $20 \%$, and the FBII would be generated by CO. As one of such cases, the FBII with the coherent beam oscillation frequency of about 7.0 MHz is generated due to $\mathrm{CO}$ at $196.0 \mathrm{~mA}, 1.2 \mathrm{nTorr}$ local vacuum pressure as shown in Fig. 1. Note that the RMS synchrotron oscillation amplitude as well as the coherent beam oscillation amplitude are increased along the bunch train as shown in Figs. 1 and 2(a). These facts are well agreed with our past FBII experimental results in the PLS storage ring [2], [3].

Generally, after the installation of a new vacuum component, the population of $\mathrm{CO}$ is downed to $5 \%$ within one month operation of the PLS storage ring. In this case, we can not observe the FBII due to $\mathrm{CO}$. However, $\mathrm{H}_{2}$ instead of $\mathrm{CO}$ would mainly generate the FBII at the very high vacuum pressure and the high beam current. Since the lightest molecule among the several trapped ones has the shortest instability growth time, $\mathrm{H}_{2}$ would asymptotically determine the coherent beam oscillation [1]. On April 18, 2000, we observed one strong FBII due to $\mathrm{H}_{2}$ at $280.27 \mathrm{~mA}$, 70.0 nTorr local vacuum pressure as shown in Figs. 2(b) and 3. Since the population of $\mathrm{H}_{2}$ is much higher than those of other molecules, and the local vacuum pressure as well as the population of $\mathrm{H}_{2}$ is increased by about four times at near 6:09, the bump of 70.0 nTorr in the vacuum pressure may be mainly due to $\mathrm{H}_{2}$. We can clearly ob-

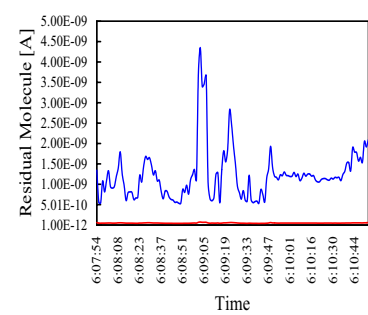

(a)

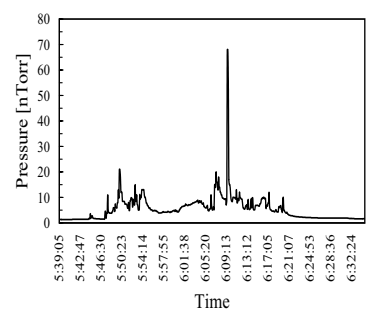

(b)
Figure 3: Residual molecule population of $\mathrm{H}_{2}$ (top) and others (bottom, close to the horizontal axis) (a) and the local vacuum pressure (b) in the PLS vacuum chamber at near $280.27 \mathrm{~mA}, 2.04 \mathrm{GeV}$ on April 18, 2000.

serve the RMS synchrotron oscillation amplitude increase along the bunch train and some saturation or slow down of the RMS synchrotron oscillation amplitude at the tail part of the train as shown in Fig. 2(b). This saturation can be generated when the vertical oscillation amplitude of the beam centroid is higher than about one RMS vertical beam size, $\sigma_{y} \simeq 90.0 \mu \mathrm{m}$, due to the nonlinearity of the beam-ion force and the detuning of the ion oscillation frequency during the strong vertical beam shaking [1], [3]. Since it is difficult to measure the vertical beam size clearly because of the vertical beam shaking, we can not calculate the ion oscillation frequency exactly with the linear theory [1]. However, there are broadband oscillation spectra around 19.2 MHz which is the ion oscillation frequency due to $\mathrm{H}_{2}$ when the RMS horizontal and vertical beam sizes are about $389.7 \mu \mathrm{m}$ and $89.8 \mu \mathrm{m}$, respectively, at $280.27 \mathrm{~mA}$.

\section{LONGITUDINAL DAMPING}

At near 6:09, April 18, 2000, by the help of the LFS DAS, we have simultaneously recorded the time domain 400-bunch synchrotron oscillation amplitude as shown in Fig. 4(a) when the $\mathrm{H}_{2}$ induced FBII is generated. Note that the time domain 400-bunch synchrotron oscillation amplitude is substantially damped down at near $\tau_{c}=15 \mathrm{~ms}$, the elapsed time after the LFS recording. By taking the turn-by-turn fast Fourier transform (FFT) of the time domain beam motions, we can obtain the time evolution of one longitudinal CBMI with the mode number $n=194$ due to the 1707.0 MHz higher order mode (HOM) of the RF cavities as shown in Fig. 4(b). Note its modal strength is also substantially damped down at near $\tau_{c}$. By exponentially fitting the CBMI evolution in Fig. 4(b), the FBII driven longitudinal damping time can be measured as about $2.50 \mathrm{~ms}$. Here, we have considered the synchrotron radiation damping time of $7.84 \mathrm{~ms}$ at $2.04 \mathrm{GeV}$, and the CBMI growth time of $2.47 \mathrm{~ms}$ at $287.27 \mathrm{~mA}, 2.04 \mathrm{GeV}$. By reminding that the damping time of the normal active LFS is about $1 \sim 2 \mathrm{~ms}$, the FBII can drive a very strong longitudinal damping mechanism under the synchro-betatron 


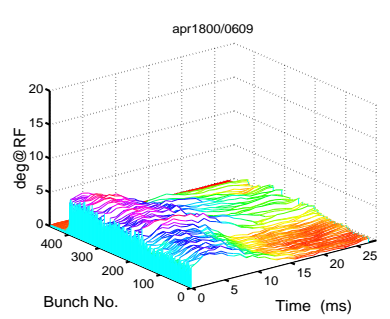

(a)

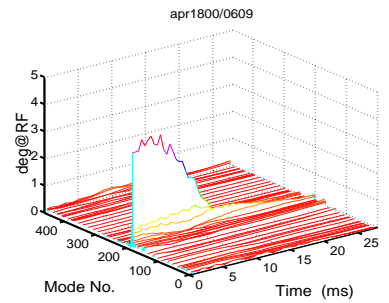

(b)
Figure 4: Time domain 400-bunch synchrotron oscillation amplitude (a) and its evolution of a CBMI with the mode number of 194 (b) immediately after the strong vertical beam shaking is generated due to $\mathrm{H}_{2}$ induced FBII at $280.27 \mathrm{~mA}, 70.0$ nTorr.

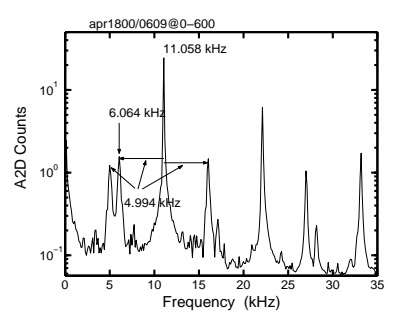

(a)

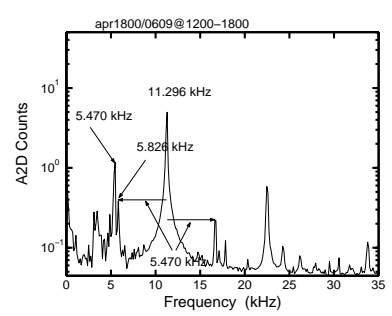

(b)
Figure 5: Beam signal spectra before $\tau_{c}$ (a) and after $\tau_{c}$ (b) when the $\mathrm{H}_{2}$ induced FBII is generated at $280.27 \mathrm{~mA}$, 70.0 nTorr.

coupling status.

To analyze the FBII driven longitudinal damping, we have investigated the beam signal spectra from the LFS raw data as shown in Fig. 5. Before $\tau_{c}$, there is one RF noise with the first harmonic frequency of $4.994 \mathrm{kHz}$ as shown in Fig. 5(a). Note that there are $4.994 \mathrm{kHz}$ satellites around the synchrotron frequency of $11.058 \mathrm{kHz}$, which indicates that the beams are mainly modulated by the first RF noise harmonic. Since the RF noise in the PLS storage ring is generated due to the large phase offset at the front of the phase detector in the phase loop of the RF station, the noise frequency can be changed under a sudden beam shaking. Actually, after $\tau_{c}$, the synchrotron frequency and the noise frequency are changed to $11.296 \mathrm{kHz}$ and $5.470 \mathrm{kHz}$, respectively, as shown in Fig. 5(b) due to the FBII under the synchro-betatron coupling. Although the amplitude of the first harmonic is almost constant for two cases, the satellites around the synchrotron frequency or the modulation strength due to the first noise harmonic is substantially reduced as shown in Fig. 5(b). This means that the main modulation frequency is changed from the first harmonic frequency to the second one which is close to the synchrotron frequency. As described in the reference [5], the RF noise has several harmonics. However, due to the limited bandwidth of about $11 \mathrm{kHz}$ from the low level RF system to the RF cavity, only two or three noise harmonics whose frequencies are within the band- width can affect the beam motions via the RF phase modulation. The various beam properties such as the beam instability or the bunch length can be nonlinearly changed by only one RF noise harmonic near the bifurcation frequency which is a little smaller value of the synchrotron frequency [5]. Note that there is the second noise harmonic below the high peak of the synchrotron frequency even though we can not clearly see it due to the large amplitude of synchrotron frequency. After $\tau_{c}$, the difference between the second noise harmonic frequency of $5.470 \times 2=10.94 \mathrm{kHz}$ and the synchrotron frequency of $11.296 \mathrm{kHz}$ is about $356 \mathrm{~Hz}$ while it is about $1070 \mathrm{~Hz}$ before $\tau_{c}$. Therefore, after $\tau_{c}$, the strength of the dipole mode parametric resonance of the RF phase modulation due to the second noise harmonic becomes stronger than before $\tau_{c}$ [5]. According to the simulation, as the modulation frequency approaches the bifurcation frequency from below such as after $\tau_{c}$, one outer stable fixed point and one unstable fixed point will move in and one inner stable fixed point will move out [5]. In this case, the synchrotron oscillation amplitude around two stable fixed points will be reduced, and the net dipole mode phase oscillations in the bunches which invoke the CBMI can be substantially reduced by the dipole mode parametric resonance [5].

\section{SUMMARY}

The vertical beam shaking due to the FBII changes the synchrotron oscillation frequency and the RF noise frequency via the synchro-betatron coupling. After these frequency changes, the strength of the dipole mode parametric resonance due to the second noise harmonic is increased. With the combined action of the FBII, the synchro-betatron coupling, and the parametric resonance due to RF noise, one longitudinal CBMI due to the 1707.0 MHz HOM can be substantially damped. The small transverse beam sizes and stable beam motion were preserved for a while due to this damping even though the beam current is high.

\section{REFERENCES}

[1] T. O. Raubenheimer and F. Zimmermann, Phys. Rev. E 52, 5487 (1995).

[2] M. Kwon et al., Phys. Rev. E 57, 6016 (1998).

[3] J. Y. Huang et al., Phys. Rev. Lett. 81, 4388 (1998).

[4] J. Byrd et al., Phys. Rev. Lett. 79, 79 (1997).

[5] Yujong Kim et al., "RF Noise Driven Dipole Mode Parametric Resonance," these proceedings.

[6] R. Baartman and U. Wienands, TRIUMF Report No. TRIPP-90-29, 1990, T. Suzuki, KEK Preprint No. 84-21, 1985. 